\title{
Avant-propos de la Première Édition
}

De nos jours, il n'est guère de branche de la physique que l'on puisse sérieusement aborder sans une bonne connaissance de la Mécanique Quantique. L'exposé qui en est donné dans ce traité est, je l'espère, suffisamment simple pour être accessible aux étudiants et cependant suffisamment complet pour pouvoir servir d'outil de travail aux physiciens confirmés.

Ce livre est issu d'un cours professé au Centre d'Études Nucléaires de Saclay depuis 1953. De nombreuses discussions, tant avec les étudiants qu'avec mes collègues, m'ont considérablement aidé à en clarifier la présentation. Plusieurs personnes, à qui j'avais communiqué certaines parties du manuscrit, ont bien voulu me faire part de leurs critiques; parmi elles, je voudrais citer MM. Edmond Bauer et Jean Ullmo, auxquels je dois d'intéressantes remarques concernant l'exposé des principes. Je suis particulièrement reconnaissant à $M$. Roger Balian de s'être livré à l'examen critique d'une grande partie du manuscrit et de m'avoir suggéré de nombreuses améliorations ; qu'il veuille bien trouver ici l'expression de ma profonde gratitude. Enfin, je tiens à remercier ceux de mes étudiants qui ont bien voulu vérifier le texte et les calculs des différents chapitres et m'aider dans la correction des épreuves.

Les problèmes qui figurent à la fin de chaque chapitre n'ont pas seulement été choisis pour leur valeur éducative, mais aussi pour signaler certaines propriétés dignes d'intérêt; cela explique la difficulté relative de certains d'entre eux.

Les quelques ouvrages ou articles cités en référence ont pour objet d'aider le lecteur à compléter ou approfondir certains passages. Il était hors de question de donner une bibliographie complète des différents sujets traités. Un volume entier n'y aurait pas suffi.

Albert Messiah, Octobre 1958. 
